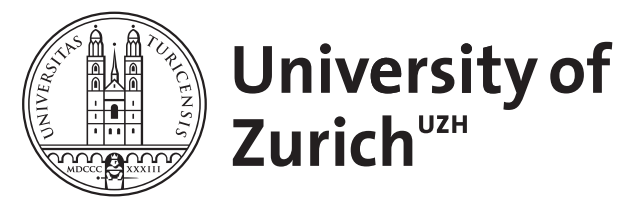

\title{
What can we learn from epigenetics in the year 2009 ?
}

\author{
Jüngel, A ; Ospelt, C ; Gay, S
}

\begin{abstract}
PURPOSE OF REVIEW: Rheumatoid arthritis (RA) is a systemic, autoimmune disease resulting in the destruction of affected joints. Even though current therapies with biologics such as tumor necrosis factor-alpha blockers yield significant improvement for the patients, the disease is not curable yet. Therefore, we need novel strategies for better therapies. RECENT FINDINGS: The growing knowledge of epigenetics might give us new insights into the pathogenesis of autoimmune diseases. In the last year, several new findings about epigenetic modifications of gene expression were reported in different arthritides. These modifications describe changes in the expression of DNA that result from methylation, posttranslational modifications of the histone proteins, including acetylation/deacetylation, sumoylation, methylation and microRNAs. Most interestingly, these modifications seem to act in concert and are associated with the circadian metabolic rhythm of cells. SUMMARY: This review summarizes reports from the last year about epigenetic modifications of gene expression via acetylation/deacetylation, including sirtuins, sumoylation, methylation, microRNAs in all in rheumatoid arthritis and other arthritides, providing potential strategies for better therapies and encourages the development of specific epigenetic drugs.
\end{abstract}

DOI: https://doi.org/10.1097/BOR.0b013e3283389641

Posted at the Zurich Open Repository and Archive, University of Zurich

ZORA URL: https://doi.org/10.5167/uzh-38515

Journal Article

Accepted Version

Originally published at:

Jüngel, A; Ospelt, C; Gay, S (2010). What can we learn from epigenetics in the year 2009? Current Opinion in Rheumatology, 22(3):284-292.

DOI: https://doi.org/10.1097/BOR.0b013e3283389641 


\section{Review Current Opinion Rheumatology}

\section{Title}

What can we learn from epigenetics in rheumatoid arthritis in the year?

Astrid Jüngel, Caroline Ospelt, Steffen Gay

Center of Experimental Rheumatology, University Hospital and Zurich Center of Integrative Human Physiology (ZIHP), Gloriastrasse 23-25, Zurich, Switzerland

Address correspondence to:

Astrid Jüngel, PhD

Center of Experimental Rheumatology,

University Hospital Zurich, Gloriastrasse 23-25,

CH- 8091 Zurich, Switzerland

Phone: +41 44255 3729; Fax: +41-44-255-4170;

E-mail: Astrid.Juengel@usz.ch 


\section{Abstract}

\section{Purpose of review}

Rheumatoid arthritis (RA) is a systemic, autoimmune disease resulting in the destruction of affected joints. Even though current therapies with biologics such as TNF- $\alpha$ blockers yield significant improvement for the patients, the disease is not curable yet. Therefore, we need novel strategies for better therapies.

\section{Recent finding}

The growing knowledge of epigenetics might give us new insights into the pathogenesis of autoimmune diseases. In the last year several new findings about epigenetic modifications of gene expression were reported in different arthritides. These modifications describe changes in the expression of DNA that result from methylation, posttranslational modifications of the histone proteins including acetylation/deacetylation, sumoylation, methylation and microRNAs. Most interestingly, these modifications seem to act in concert and are associated with the cirardian metabolic rhythm of cells.

\section{Summary}

This review summarizes reports from the last year about epigenetic modifications of gene expression via acetylation/deacetylation, including sirtuins, sumoylation, methylation and/or microRNAs in RA and other arthritides, providing potential strategies for better therapies and encourages the development of specific epigenetic drugs. 
Keywords (5)

Epigenetics, HDACs, sirtuins, sumoylation, methylation, microRNA, Rheumatoid arthritis 


\section{Introduction:}

We summarize here reports from the last year about epigenetic modifications that control the regulation of gene expression in autoimmune diseases. Epigenetic modifications of synovial cells such as fibroblasts, chondroyctes and endothelial cells and also from PBMCs were reported in different arthritides. This knowledge gives us novel insights into the pathogenesis of these diseases and allows in future the development of more specific epigenetic drugs.

\section{Text of review:}

\section{HDACs and sirtuins}

Protein acetylation is the best described posttranslational modification of histones that is able to regulate the transcription of genes. Acetylation of histones is mediated by a group of enzymes so called histone-acetyltransferases (HATs) that transfer acetyl groups from acetyl-coenzyme $A$ to the $\varepsilon$-amino group of lysines. HATs are counterbalanced by the activity of histone-deacetylases (HDACs). HDACs are divided into three classes based on their homologies with the yeast transcriptional repressors: Rpd3p (reduced potassium dependency gene 3) belonging to class I (HDAC1, 2, 3, 8 and 11); Hda1p (a subunit of histone deacetylase A complex) belonging to class II (HDAC4, 5, 6, 7, 9, and 10); and Sir2p (silent information regulator 2) belonging to class III (Sirtuins 1 to 7 ) (1, 2). In 2009, a report about the altered distribution pattern of HDACs on chromatin, the dissociation of the deacetylase activity from the DNA binding sites and the function of gene repression provided novel insights into the function of chromatin remodeling enzymes. In addition, HDACs themselves are also subject to post-translational modifications that 
influence stability, localization, activity and non-histone protein-protein interactions on the DNA binding sites (3). Several novel targets of HDACs isoforms such as transcription factors are described recently. This knowledge may provide us better therapeutically strategies in RA $(4,5)$ and other arthritides by targeting the catalytic activity of HDACs, their DNA binding regions and/or their post translational modifications (6).

To control the acetylation status in the cells HATs and HDACs work antagonistically. The removal of the positive charge from the histone protein during acetylation is commonly associated with active gene transcription whereas deacetylation is associated with gene repression, indicating that acetylation and deacetylation are highly dynamic processes. Recently, Wang et al reported a novel distribution pattern for these enzymes by mapping the genome-wide distribution of HATs and HDACs in human primary resting $\mathrm{CD} 4^{+} \mathrm{T}$ cells. They showed that both- HATs and HDACs are primarily targeted to transcribed regions of active genes (7). They show two major functions for the accumulation of HDACs on active gene regions. First, the HDACs work to reset chromatin by removing the acetylation marks. And secondly, HDACs have a function in H3K4 primed genes, where transient binding of HATs and HDACs prevents RNA polymerase II (Pol II) from binding and keeps the promoter of a gene in an inactive state. Totally silent genes which are associated with H3K27 methylation signals did not show detectable levels of acetylation and deacetylation activities. These results help us to understand how chromatin remodeling enzymes work in the transcriptional regulation. However, the specific repression of genes by HDAC isoforms, their binding sites on DNA and interactions with other DNA binding molecules is not known yet.

HDACs have been reported to be included in many cellular pathways and their dysreguation has been linked to multiple cancers as well as to autoimmune diseases 
(8-12). Our group reported a reduction of total HDAC activity in the synovial tissue of patients with RA and a significant downregulation of the isoform proteins HDAC1 and HDAC2 suggesting that HDACs are intrinsically reduced and therefore might not be suitable for further treatment by HDAC inhibitors (HDACi) (13). However, in several animal studies HDACi showed beneficial effects for treatment of arthritis (14-16). These results show that further knowledge of HDACi on histones and non-histone proteins in different cell populations is necessary before these drugs can be safely used for the treatment of RA (Table 1).

To understand the function of acetylases/deacetylases in regard to the production of matrix metalloproteinases (MMPs) we established their expression pattern in the synovial fibroblasts (RASF) which are the effector cells leading to cartilage destruction in RA (17). In contrast to synovial tissues, we could not detect a decreased expression of HDAC1 and 2 in TNF- $\alpha$ and IL-1 $\beta$ stimulated RA synovial fibroblasts, and therefore these HDACs do not contribute to the increased activity of acetylation seen in the tissue. But, stimulation of the RASF with TNF- $\alpha$ and IL-1 $\beta$ to mimic the inflammatory milieu in the affects joints resulted in an increase of the global acetylation status of the cells and reduced expression of the isoforms HDAC 3, 4 and 7 (18). Treatment of the cells with the HDACi trichostatin A resulted in a significant downregulation of HDAC7 (19). In addition, TSA also downregulated the expression of MMPs in stimulated cells, while their expression in unstimulated cells was unaffected, suggesting an interaction of HDACs with molecules from the inflammatory pathway (20). Silencing of HDAC7 by siRNA resulted in reduced expression of MMP-1 and 3 implying that specific targeting of HDAC7 could be a strategy for anti-destructive therapy. In contrast, Horiuchi $\mathrm{H}$ et al reported a significant overexpression of HDAC1 in RASF compared to OASF at the mRNA and protein level. HDAC2 transcripts showed the highest mRNA expression levels of all 
HDACs in RA and OASF. Knock down of HDAC1 and HDAC2 by siRNA in RASF resulted in significantly reduced cell viability even in the presence of a strong growth factor such as plateled-derived growth factor (PDGF), upregulated expression of the cell cycle proteins p16 and p21 and p53, and increased TUNEL positive cells. Knockdown of HDAC1 in addition upregulated TNF- $\alpha$ induced MMP-1 production by RASF. Therefore, these HDAC isoforms induce proliferation, reduce apoptosis and play a role in inhibiting MMPs (21). Since acetylation and deacetylation are highly dynamic processes influenced by the stage of development, different expression patterns of HDACs might result from the use of different passages of cultured synovial fibroblasts from patients with RA and OA. Our group only used cells from passages 4 to 6 to make sure that the isolated cells were a pure culture of fibroblasts. Horiuchi $M$ et al used RASF as early as from passage 2 to 5 and OASF from passage 1 to 2 for their experiments.

A misregulation of HDAC isoforms was also reported in other arthritides. Overexpression of HDAC1 and 2 proteins was demonstrated in human chondrocytes from OA cartilage consistent with a downregulation of cartilage marker genes. Overexpression of these HDACs repressed specific cartilage marker genes such as aggrecan and collagen 2 and to a different extent also collagen 9 , collagen 11 , dermatopontin and cartilage oligomeric protein (COMP) whereas silencing of HDAC1 and 2 by siRNA or HDACi increased cartilage gene expression. Removal or exchange of one of the DNA binding sites, the carboxy-terminal domain (CTDs), showed altered target gene repression specificity for HDAC1 and HDAC2. The most interesting point of this study is that these CTDs can function independently of the HDAC enzymatic activity to target HDACs to specific genes (22). Others reported elevated levels of the isoform, HDAC7 in OA cartilage compared to healthy donors. Silencing of HDAC7 in a human chondrosarcome cell line suppressed the IL-1 
induced expression of MMP13 suggesting that specific HDAC7 suppression may prevent the increased matrix degradation in $\mathrm{OA}(23)$.

In another study, HDAC inhibitors were shown to antagonize FGF2 and IL-1 induced expression of MMP1, 3 and 13 in cultured human articular chondrocytes, also suggesting HDAC inhibition as intervention in OA. But further analysis showed that also cartilage matrix proteins such as COL2A1 and aggrecan were also reduced by the treatment with TSA. Whether HDACi may slow down cartilage turnover and thereby inhibit the progression of cartilage destruction to preserve existing cartilage needs to be evaluated in animal models (24).

Together, the mentioned studies suggest that HDACs might have several functions and targets, namely the deacetylase activity on histone proteins and non-histone proteins and repression of gene expression by binding to promoter regions alone or in complex with other DNA binding molecules. Therefore, it is very important to explore all the possible effects of HDAC isoforms before using them for treatment.

Based on the evidence that epigenetic modifications of gene regulation contribute also to the angiogenic function of endothelial cells Urbich et al analyzed the function of histone deacetylase isoforms in angiogenesis. This study is of interest because angiogenesis plays an important role in RA where inflammation and subsequent joint destruction in the synovium are also dependent on the development of new vasculature. They showed that HDAC5 is a negative regulator of angiogenesis in endothelial cells by binding to the promoter and repressing FGF2 and Slit2. Knock down of HDAC5 lead to an increased endothelial cell migration, sprouting, and tube formation whereas overexpression of HDAC5 decreased sprout formation. In contrast, silencing of other histone deacetylases such as HDAC7 and HDAC9 blocked angiogenesis (25). The function of HDAC7 in endothelial cells has been attributed to the repression of the transcription factor MEF2 resulting in the inhibition 
of MMP-10 (26) whereas the proangiogenic function of HDAC9 was not described before. Of interest is that the repressive function of HDAC5 was independent of the direct deacetylase activity of HDAC5 suggesting an interaction with other DNA binding molecules or other HDAC isoforms as it is described for class II HDACs. However, the deacetylase- deficient HDAC5 mutant which lacks also the C-terminus still repressed endothelial function, implying other mechanism for the antiangiogenic effects. The authors suggest that the N-terminal part of HDAC5 might also repress transcriptional activity through interaction with co-repressors such as HP1 or CtBP.

In RA HDAC inhibitors were also used to alter gene expression in hematopoietic cells. In RA, regulatory $T$ cell function is defective because they are unable to prevent the release of inflammatory cytokines from effector $\mathrm{CD} 4^{+} \mathrm{CD} 25^{-} \mathrm{T}$ cells, they do not suppress effector T cell proliferation, and they show low levels of FOXP3 expression. Saouaf et al showed that treatment with the HDACi valproic acid (VPA, i.p., 400 $\mathrm{mg} / \mathrm{kg}$ ) increased regulatory $\mathrm{T}$ cell function and decreased the incidence and severity of collagen-induced arthritis. They could observe that VPA increased the suppressive function of $\mathrm{CD}^{+} \mathrm{CD} 25^{+}$Tregs and the number of $\mathrm{CD} 25^{+} \mathrm{FOXP} 3^{+}$Tregs in vivo (27, 28).

Of interest for the pathogenesis in $\operatorname{RA}(29,30)$ was also the report from Villagra et al showing that histone deacetylases regulate the expression of genes involved in inflammatory responses. They showed that HDAC11 interacts with the transcription activators STAT3 or Sp1 on the promoter of the gene encoding interleukin 10 and negatively regulated the expression of this cytokine in mouse and human antigenpresenting cells (APCs) (31). Overexpression of HDAC11 in APCs activated naïve antigen-specific CD4 ${ }^{+} \mathrm{T}$ cells whereas downgregulation of HDAC11 impaired antigenspecific $\mathrm{CD}^{+} \mathrm{T}$ cell response. In this case, gene repression of HDAC11 seems to be 
mediated by the enzymatic activity, as overexpression of an HDAC11 mutant with a deleted deacetyltransferase domain did not inhibit IL-10 expression.

Posttranslational modifications of proteins, such as citrullination, which is produced by the calcium binding peptidylarginine deiminases (PADIs) are of particular interest in the pathogenesis of $\operatorname{RA}(32,33)$. Two isoforms, PADI2 and PADI4, are overexpressed in RA and contribute to the generation of specific substrates for the anti-citrullinated protein antibodies (ACPA). In addition, PADI4 itself is a target of autoantibodies in RA. PAD4 plays a physiological role in regulation of gene expression via citrullination of arginine residues of histone proteins (H3R2, 8, 17 and 26, and H4R3 and 42), thereby antagonizing histone methylation. Denis et al reported recently a functional connection between deimination and deacetylation of histones (34). They could show that PADI4 associated with HDAC1 both in vitro and in vivo to repress gene transcription of the estrogen-regulated pS2 promotor.

Sirtuins, the class III HDACs, differ from the zinc dependent class I and class II HDACs because they require nicotinamide adenine dinucleotide (NAD ${ }^{+}$) as an essential cofactor. In addition, sirtuins also deacetylate non-histone proteins in the nucleus (SIRT1, 6, 7), in the cytoplasm (SIRT2) or in mitochondria (SIRT3, 4, 5). All 7 members of this family of which SIRT1 has become the most well studied protein are associated with the regulation of metabolism, cellular survival and aging $(35,36)$. Of interest, SIRT1-null mice suffer from a mild autoimmune condition that is apparent by the deposition of immune complexes in the liver and kidney and they develop high titer anti-nuclear antibodies. Older animals develop a sporadic a form of diabetes (37).

For RA, sirtuins might play an important role since they are also considered as mediators of inflammation. They consume NAD+ as an essential cofactor and generate nicotinamide as they hydrolytically remove an acetyl group from a lysine 
residue from the target protein. The deacetylation process includes the hydrolysis of $\mathrm{NAD}+$, yielding nicotinamide and an O-alkylamidate intermediate O-acetyl-ADPribose (OAADPr). The cofactor NAD+ directly activates sirtuins while nicotinamide as well as NAD in its reduced form NADH directly inhibit sirtuin enzyme activity. Beyond its central role in oxidative metabolism, NAD is increasingly considered to be involved in the regulation of intracellular signaling (38).

In vivo, sirtuins are tightly regulated by modulators of sirtuin activity. SIRT1 interacts and can be activated by active regulator of SIRT1 (AROS). AROS works as a derepressor by displacing the inhibitory factor deleted in breast cancer1 (DBC1). DBC1 was shown to bind and inhibit SIRT-1-catalyzed deacetylation of p53 in vivo by binding of NAD+ and OAADPr to the DBC1 complex. Resveratrol, a phytoalexin produced naturally by several plants, activates SIRT1 by displacing the inhibitory factor DBC1. The disruption of SIRT1- DBC1 binding offers novel therapeutic strategies to activate SIRT1 expression in vivo by pharmaceutical compounds (36). Recent reports show that that $\mathrm{DBC} 1$ was upregulated in cartilage of patients with $\mathrm{OA}$ suggesting SIRT1 as a regulator for gene expression in chondrocytes. Furthermore, they reported that the decreased levels and activity of SIRT1 in OA articular cartilage are associated with an increased level of matrix degrading enzymes such as MMP-3 and -13 as well as with increased apoptosis through the repression of tyrosine phosphatase 1B (PTP1B) (39).

Other reports show an association between the circadian clock and the cellular metabolism which is also of interest for RA (40). The transcriptional regulators CLOCK and BMAL1 dimerize and bind to $E$ box enhancer sequences in the genes for period (per) and cryptochrome (cry). The expression of CRY and PER proteins subsequently leads to the repression of CLOCK: BMAL1 transcripts suggesting a translational-transcriptional feedback mechanism (41). Of interest, TNF- $\alpha$ inhibits 
CLOCK-BMAL1-induced activation of gene promotors suggesting that the proinflammatory milieu in autoimmune diseases impairs clock gene functions and causes fatigue in RA patients (42). Hashiramoto et al showed a disturbed circadian rhythmic expression of PER in the nuclei of inflammatory synovial cells, disturbed expression of BMAL1 in spleen cells after induction of experimental arthritis in mice. (43). Deletion of Cry in this model resulted in an increased number of activated T cells and induced the expression of TNF- $\alpha$ in the spleen. Induced arthritis in Cry1/Cry2- knockout animals showed worsening of joint swelling, and upregulation of TNF- $\alpha$, IL-1 $\beta$ and IL-6, MMP-3, Wee-1 and c-Fos whereas ectopoic expression of Cry1 significantly reduced the activation of TNF-a gene suggesting biological clock and arthritis influence each other.

Most interestingly, the circadian regulator CLOCK is a histone acetyltransferase which is counterbalanced by the deacetylase activity of SIRT1. In vivo CLOCK acetylates and activates its partner BMAL1. Both together induce the circadian expression of nicotinamide phosphoribosyltransferase (NAMPT) by binding to its Eboxes in the promoter. Nampt on the other site provides the important cofactor NAD+ for the counter player SIRT1. For the deacetylation activity, SIRT1 is also recruited to the NAMPT promoter and contributes to its repression and therefore to the the circadian synthesis of its own coenzyme. The NAMPT inhibitor FK866 significantly lowered cellular NAD+ and NAM levels and increased acetylation of the CLOCK partner BMAL1 similar to the effect of inhibition of SIRT1 $(44,45)$.

Nampt, also known as PBEF (Pre-B-cell colony-enhancing factor) or visfatin, is upregulated in the synovial tissues from patients with RA and induced by toll-like receptors signaling in isolated synovial fibroblasts (RASF) (46).

Since calorie restriction decreases NADH levels, changes in the intracellular $\mathrm{NAD+/NADH}$ ratios have also impact on sirtuin deacetylase activity in the cells. In 
addition, metabolic stress such as prolonged fasting is known to activate Nampt which regulates SIRT1-dependent activities by its capacity to provide the SIRTcofactor NAD $(38,47)$. Our group reported Nampt/PBEF not only as a novel marker of inflammation in RA with proinflammatory but also matrix-degrading activities (46). Furthermore, we could show that RNA interference-mediated gene therapy of PBEF reduces the severity of collagen-induced arthritis in mice suggesting PBEF as a crucial proinflammatory and destructive mediator of joint inflammation in vivo (48). Whereas in smooth muscle cells PBEF delays cellular senescence through SIRT1mediated deacetylation and inactivation of p53 $(47,49)$ the mechanism of PBEF regulation of sirtuins in RA is currently under investigation. Recently, our group reported the expression of all sirtuins in isolated synovial fibroblasts from patients with RA (RASF) with a significant upregulation of SIRT 1 and 4 compared with cells from osteoarthritis (OA). The expression level of single sirtuin-isoforms could be downregulated by different ligands of the Toll-like receptor (TLR) pathway whereas PBEF was up-regulated suggesting a regulatory role for sirtuins in the innate immune system via TLR signaling (50).

Another important target for SIRT1 in RA is nuclear factor-kappa B (NFKB), the master regulator of innate immune responses, regulating inflammation, cell cycle and apoptosis. SIRT1 downregulates NFKB mediated proinflammatory effects by deacetylating its subunit RelA/p65 $(51,52)$. In addition, activation of SIRT-1 resulted in inhibition of TNF- $\alpha$ and other proinflammatory molecules such as ICAM, MCP-1, RANTES and TGF- $\beta$ suggesting that SIRT1 acts through multiple downstream pathways $(35,53)$.

Sumoylation is one of the modulators of sirtuin activity. SIRT1 can be modified at Lys-734 by the addition of a SUMO-group which increases the SIRT1 deacetylation activity (54). In contrast, the recruitment of the SUMOylase SENP-1 resulted in 
desumoylation and therefore repression of SIRT1 deacetylase activity and consequently acetylation and activation of target proteins such as p53. We reported an intrinsic upregulation of SUMO in synovial fibroblasts from patients with RA leading to their increased resistance of apoptosis by SUMOylation of promyelocytic leukemia protein (PML) and recruitment of DAXX to PML nuclear bodies (NBs) (55, 56). In contrast, the induced upregulation of the SUMO-protease SENP-1 which is normally downregulated in RASF was able to reverse this effect. Since SIRT-1 is also recruited to the PML NBs in the cells, and binds and regulate p53 expression an increased SUMOylation of SIRT1 might contribute to the apoptosis resistance of RASF (57).

SIRT1 is known to regulate cell survival and apoptosis of several cells. Recently, it was shown that SIRT1 also regulates apoptosis in human chondrocytes which is a characteristic feature of OA. A reduced level of SIRT1 was found in chondrocytes from patients with OA compared to normal. Induced catabolic, mechanical and nutritional stress inhibited the expression of SIRT1 in these cells. Inhibition of SIRT1 by sirtinol and nicotinamide or siRNA increased whereas the activation of SIRT1 by resveratrol decreased the percentage of apoptotic cells. At the same time mitochondria-related signals such as Bax and Bcl-2 were increased and decreased respectively suggesting that SIRT-1 regulates apoptosis through modulation of mitochondria related signaling pathways and could be a novel target for upregulation in the therapy in OA (58).

Even though SIRT1 as a deacetylase is expected to negatively effect transcription Dvir-Ginzberg et al demonstrated that the up- or downregulation of SIRT1 in human chondrocytes by resveratrol or siRNA function as a positive/negative regulator of cartilage specific molecules such as collagen 2, collagen 9 and aggrecan. SIRT1 thereby deacetylates the cartilage specific transcription factor Sox9 and enhances 
transcription of collagen 2 in association with co-activators such as the transcription factor Sp1. Since NAD+ is provided by NAMPT as a SIRT1-cofactor, NAMPT indirectly affects cartilage-specific gene expression. This suggests an imbalance of SIRT1 and NAMPT in OA (59).

In RA angiogenesis plays an important role because inflammation and subsequent joint destruction in the synovium is also dependent on the development of new vasculature. We defined recently hypoxic areas, particularly at sites of synovial invasion into bone in arthritic joints, by EF5 staining. Hypoxia induced the overexpression of DNA binding/differentiation 2 (ID-2) in synovial fibroblasts, which leads to an increased differentiation of osteoclasts from bone marrow precursors, suggesting that hypoxia-induced ID-2 may contribute to joint destruction in RA patients by promoting synovial fibroblast-dependent osteoclastogenesis $(60,61)$. Hypoxia inducible factor (HIF)-1alpha and HIF-2alpha are transcriptional regulators that control the genes induced during hypoxia. Both are significantly overexpressed in the synovial lining and stromal cells in RA relative to normal as shown by immunohistochemistry (62). Recently, Dioum et al showed that during hypoxia HIF$2 a$ is bound and thereby deacetylated and activated by SIRT1. The SIRT1 inhibitors NAM or sirtinol reduced, and the SIRT1 activator resveratrol increased the ability of SIRT1 to augment transcription of HIF-2 target genes superoxide dismutase 2 (Sod2), vascular endothelial growth factor A (VEGF-A) and erythropoietin (Epo). Whether SIRT1 itself is activated by hypoxia is not clear yet. But the SIRT1/HIF2a complex formation as well as SIRT1 mediated deacetylation of acetylated HIF2a during hypoxia or other environmental stress offers novel therapeutic opportunities also for RA $(63,64)$.

The function of SIRT1 in the endothelium was revealed by an impaired ability of SIRT 1 mutant mice to form new vessels in response to angiogenic signals $(65,66)$. 
Although endothelial cells express all sirtuins (1 to 7 ), knock down of SIRT1 was associated with a loss of sprouting angiogenesis in vitro. Since RA is characterized by inflammation and endothelial dysfunction (67) these results suggest that SIRT1 is a novel modulator of normal endothelial function and that overexpression of SIRT1 might be vasoprotective (68).

However, we have only limited knowledge about the mechanisms that regulates the function of SIRT1. Recently, miRNA 217 was observed to be overexpressed during endothelial senescence whereas SIRT1 levels decreased at the same time. Modulation of miRNA 217 alters the senescence process in endothelial cells and affects the acetylation state of the SIRT1 targets FOXO1 and eNOS which are important factors for angiogenesis and tube formation. Therefore, microRNA217 might act as an endogenous inhibitor of SIRT1 (69).

\section{Sumoylation}

Posttranslational modification of DNA binding molecules like transcription factors by the small ubiquitin-releted modifier SUMO has been correlated with transcriptional repression. Several proteins are recruited to promotors in a SUMO-dependent manner, such as HDAC2, the histone demethylase LSD1, the histone methyltransferase SETDB1, and the chromatin associated proteins HP1 suggesting SUMO as a central player in coordinating chromatin modifications that regulate gene expression (70). Our group showed that the SUMOylation pathway regulates the production of matrix degrading enzymes by the activity of HDAC4. We showed an increase of acetylated histone 4 in the promoter of MMP-1 in RASF compared to OASF which was decreased by the overexpression of the SUMO-specific protease SENP-1. Furthermore, overexpression of SENP-1 in RASF significantly reduced the production of MMP-1 and therefore the invasiveness of RASF due to an 
accumulation of HDAC4 on the MMP-1 promotor. Interference with these epigenetic control mechanisms might be of value for anti-destructive treatment in RA (71).

\section{Methylation}

Methylation is the best analyzed posttranslational modification regulating gene expression and contributing to several diseases, in particular autoimmune diseases (72, 73). Recently, we demonstrated that the DNA in RA synovial fibroblasts is hypomethylated, resulting in the activated and aggressive phenotype of these cells (74). Our group showed also that proliferating RASFs are deficient in DNA methyltransferase 1 (DNMT1) compared to OASF and the DNA showed fewer 5methylcytosine and methylated CG marks upstream of an L1 open-reading frame. Moreover, using 5-azacytydine, a potent inhibitor for DNMT1, on healthy fibroblasts the activated phenotype could be induced by upregulation of genes, including growth factors and receptors, extracellular matrix proteins, adhesion molecules, and matrixdegrading enzymes. These data suggest that DNA hypomethylation contributes to the chronicity of RA and could be responsible for the limitation of current therapies (75).

Furthermore, downregulation of DNMTs seems not only to affect fibroblasts but also has consequences for hematopoietic cells. Liu et al reported that decreased DNMT levels contribute to abnormal gene expression in senescent $\mathrm{CD} 4^{+} \mathrm{CD} 28^{-} \mathrm{T}$ cells (76). This is of interest for RA since chronic stimulation of CD4+ T cells in vitro results in the development of a senescent $\mathrm{CD}^{-} 8^{-}$subpopulation.

Novel therapeutic intervention using microRNAs might be also of value for RA since the expression of DNMTs themselves are regulated by specific microRNAs as recently reported (77). Garzon et al identified binding sites for miR-29b within the 


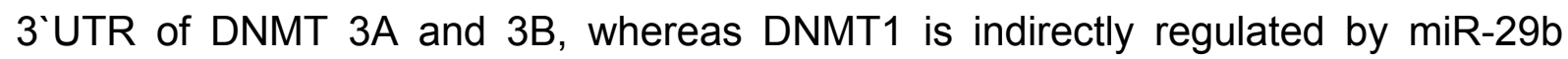
which targets Sp1, a transactivator of the DNMT1 gene (78). The enforced expression of miR-29b in leukemia cells resulted in a marked reduction of the expression of DNMT1, 3A, and 3B, suggesting a novel functional link between miRNAs and aberrant DNA hypermethylation.

\section{MicroRNA}

MicroRNAs are small RNA molecules that can negatively control their target gene expression posttranscriptionally. They play a key role in all physiological processes by direct targeting or modulating chromatin structure. Recent reports show that microRNAs can regulate epigenetic modifications but can also be a target of epigenetic modifications (79). In 2009 several studies reported about altered expression patterns of microRNAs in synovial cells from patients with RA (80-85).

Our group reported first an increased expression of microRNA-155 and -146 in RA and their involvement in the modulation of the agressive phenotype of RASF (80). In addition, we could demonstrate that miRNA-203 is overexpressed and involved in the regulation of IL-6 and MMP-1 in RASF, which contributes to their activated phenotype. Most interestingly, we could show a stimulatory effect of the hypomethylating drug 5-azacytidine on the expression of miRNA-203 in healthy fibroblasts suggesting that epigenetic mechanisms interact with altered microRNA levels in these cells (82).

Further, downregulated levels of miR-124a were reported in synovial fibroblasts from patients with RA (83). Nakamachi et al showed that transfection of precursor miRNA124a inhibits the proliferation of RASF via binding to cyclin-dependent kinase 2 (CDK-2) and monocyte chemoattractant protein 1 (MCP-1) mRNA. Overexpression of miRNA- 124a in RASF suppressed the production of CDK-2 and MCP-1 protein 
whereas overexpression of other miRNAs such as pre-miR-146a, pre-miR-223 in OASF did not induce any cytokine or chemokine expression in these cells.

Alsaleh et al showed that RASF treated with LPS express mRNA but no protein for IL-18 due to an increased degradation of the transcripts. At the same time they observed an upregulation of microRNA-346 in these cells. Suppression of this microRNA resulted in the expression of IL-18 protein by indirect regulation of the LPS-induced Bruton`s tyrosine kinase, which is typically involved in the stabilization of cytokine mRNAs (84). These results show first that cytokine secretion can be negatively regulated at the posttranscriptional level by microRNAs and secondly, that microRNAs can be induced by ligands of the innate immune system and can act as potential negative regulators of inflammation.

Most interestingly, Nagata et al have proven for the first time beneficial effects of in vivo administered microRNAs. They demonstrated the induction of apoptosis via downregulation of $\mathrm{Bcl}-2$ in the synovium of mice with autoantibody-mediated arthritis by the intraacticular injection of double stranded microRNA-15a (85). These results might be used for novel therapeutical strategies inducing the overexpression of microRNAs in vivo by intraarticular injection of double stranded miRNA into the joints.

\section{Conclusion:}

In summary, the reported data clearly demonstrate that the epigenetic modulations observed in health and disease are regulated by novel mechanisms including acetylation, methylation, sumoylation and microRNA. In this regard a complete new class of drugs is evolving (6). 


\section{References}

1. Huber LC, Stanczyk J, Jungel A, et al. Epigenetics in inflammatory rheumatic diseases. Arthritis Rheum. 2007 Nov;56(11):3523-31.

2. Kwon HS, Ott M. The ups and downs of SIRT1. Trends Biochem Sci. 2008 Nov;33(11):517-25.

3. Brandl A, Heinzel $\mathrm{T}$, Kramer $\mathrm{OH}$. Histone deacetylases: salesmen and customers in the post-translational modification market. Biol Cell. 2009 Apr;101(4):193-205.

4. Klareskog L, Catrina Al, Paget S. Rheumatoid arthritis. Lancet. 2009 Feb 21;373(9664):659-72.

5. Taylor PC, Feldmann M. Anti-TNF biologic agents: still the therapy of choice for rheumatoid arthritis. Nat Rev Rheumatol. 2009 Oct;5(10):578-82.

6. Karberg S. Switching on epigenetic therapy. Cell. 2009 Dec 11;139(6):102931.

7. Wang Z, Zang C, Cui K, et al. Genome-wide mapping of HATs and HDACs reveals distinct functions in active and inactive genes. Cell. 2009 Sep 4;138(5):101931.

8. Dieker J, Muller S. Epigenetic histone code and autoimmunity. Clin Rev Allergy Immunol. 2009 Aug 7.

9. van der Maarel SM. Epigenetic mechanisms in health and disease. Ann Rheum Dis. 2008 Dec;67 Suppl 3:iii97-100.

10. Hewagama A, Richardson B. The genetics and epigenetics of autoimmune diseases. J Autoimmun. 2009 Aug;33(1):3-11.

11. Trenkmann M, Brock M, Ospelt C, et al. Epigenetics in Rheumatoid Arthritis. Clin Rev Allergy Immunol. 2009 Aug 27;Epub ahead of print. 
12. Hirst M, Marra MA. Epigenetics and human disease. Int J Biochem Cell Biol. 2009 Jan;41(1):136-46.

13. Huber LC, Brock M, Hemmatazad $\mathrm{H}$, et al. Histone deacetylase/acetylase activity in total synovial tissue derived from rheumatoid arthritis and osteoarthritis patients. Arthritis Rheum. 2007 Apr;56(4):1087-93.

14. Chung $\mathrm{YL}$, Lee $\mathrm{MY}$, Wang $\mathrm{AJ}$, et al. A therapeutic strategy uses histone deacetylase inhibitors to modulate the expression of genes involved in the pathogenesis of rheumatoid arthritis. Mol Ther. 2003 Nov;8(5):707-17.

15. Nishida K, Komiyama T, Miyazawa S, et al. Histone deacetylase inhibitor suppression of autoantibody-mediated arthritis in mice via regulation of p16INK4a and p21(WAF1/Cip1) expression. Arthritis Rheum. 2004 Oct;50(10):3365-76.

16. Nasu Y, Nishida K, Miyazawa S, et al. Trichostatin A, a histone deacetylase inhibitor, suppresses synovial inflammation and subsequent cartilage destruction in a collagen antibody-induced arthritis mouse model. Osteoarthritis Cartilage. 2008 Jun;16(6):723-32.

17. Hemmatazad $\mathrm{H}$, Maciejewska Rodrigues $\mathrm{H}$, Huber LC, et al. Anti-destructive effects of Trichostatin A (TSA) are partly mediated through altered expression of histone deacetylase 7 (HDAC7), Arthritis Rheum. 2009;60, 10, S274.

18. Hemmatazad H, Maciejewska-Rodrigues H, Gay RE, et al. Inflammatory cytokines induce global chromatin acetylation in RASF. Arthritis Rheum. 2008;58(9):S911.

19. Dokmanovic M, Perez G, Xu W, et al. Histone deacetylase inhibitors selectively suppress expression of HDAC7. Mol Cancer Ther. 2007 Sep;6(9):252534.

20. Halili MA, Andrews MR, Sweet MJ, et al. Histone deacetylase inhibitors in inflammatory disease. Curr Top Med Chem. 2009;9(3):309-19. 
21. Horiuchi M, Morinobu A, Chin T, et al. Expression and function of histone deacetylases in rheumatoid arthritis synovial fibroblasts. J Rheumatol. 2009 Jun 16.

22. Hong S, Derfoul A, Pereira-Mouries L, et al. A novel domain in histone deacetylase 1 and 2 mediates repression of cartilage-specific genes in human chondrocytes. Faseb J. 2009 Oct;23(10):3539-52.

23. Higashiyama R, Miyaki S, Yamashita S, et al. Correlation between MMP-13 and HDAC7 expression in human knee osteoarthritis. Mod Rheumatol. 2009 Sep 26.

24. Wang $\mathrm{X}$, Song $\mathrm{Y}$, Jacobi JL, et al. Inhibition of histone deacetylases antagonized FGF2 and IL-1beta effects on MMP expression in human articular chondrocytes. Growth Factors. 2009 Feb;27(1):40-9.

25. Urbich C, Rossig L, Kaluza D, et al. HDAC5 is a repressor of angiogenesis and determines the angiogenic gene expression pattern of endothelial cells. Blood. 2009 Apr 7.

26. Chang S, Young BD, Li S, et al. Histone deacetylase 7 maintains vascular integrity by repressing matrix metalloproteinase 10. Cell. 2006 Jul 28;126(2):321-34. 27. Saouaf SJ, Li B, Zhang G, et al. Deacetylase inhibition increases regulatory T cell function and decreases incidence and severity of collagen-induced arthritis. Exp Mol Pathol. 2009 Oct;87(2):99-104.

28. Wang L, de Zoeten EF, Greene MI, et al. Immunomodulatory effects of deacetylase inhibitors: therapeutic targeting of FOXP3+ regulatory T cells. Nat Rev Drug Discov. 2009 Dec;8(12):969-81.

29. Neidhart M, Jungel A, Ospelt C, et al. Deficient expression of interleukin-10 receptor alpha chain in rheumatoid arthritis synovium: limitation of animal models of inflammation. Arthritis Rheum. 2005 Oct;52(10):3315-8. 
30. Schulze-Tanzil G, Zreiqat H, Sabat R, et al. Interleukin-10 and Articular Cartilage: Experimental Therapeutical Approaches in Cartilage Disorders. Curr Gene Ther. 2009 Aug 1;Epub ahead of print.

31. Villagra A, Cheng F, Wang HW, et al. The histone deacetylase HDAC11 regulates the expression of interleukin 10 and immune tolerance. Nat Immunol. 2009 Jan;10(1):92-100.

32. Migliorini P, Pratesi F, Tommasi C, et al. The immune response to citrullinated antigens in autoimmune diseases. Autoimmun Rev. 2005 Nov;4(8):561-4.

33. Anzilotti C, Pratesi F, Tommasi C, et al. Peptidylarginine deiminase 4 and citrullination in health and disease. Autoimmun Rev. 2009 Jun 18;Epub ahead of print.

34. Denis $H$, Deplus $R$, Putmans $P$, et al. Functional connection between deimination and deacetylation of histones. Mol Cell Biol. 2009 Sep;29(18):4982-93.

35. Lavu S, Boss O, Elliott PJ, et al. Sirtuins--novel therapeutic targets to treat age-associated diseases. Nat Rev Drug Discov. 2008 Oct;7(10):841-53.

36. Smith BC, Hallows WC, Denu JM. Mechanisms and molecular probes of sirtuins. Chem Biol. 2008 Oct 20;15(10):1002-13.

37. Sequeira J, Boily G, Bazinet S, et al. sirt1-null mice develop an autoimmunelike condition. Exp Cell Res. 2008 Oct 1;314(16):3069-74.

38. Chaudhary N, Pfluger PT. Metabolic benefits from Sirt1 and Sirt1 activators. Curr Opin Clin Nutr Metab Care. 2009 Jul;12(4):431-7.

39. Gabay $\mathrm{OH}$, Gagarina V, Lee EJ, et al. Decreased levels of Sirt1 in OA articular cartilage are associated with an increase in MMP-3, -13 and apoptosis. Arthritis Rheum. 2009;60, 10, S488.

40. Sahar S, Sassone-Corsi P. Metabolism and cancer: the circadian clock connection. Nat Rev Cancer. 2009 Dec;9(12):886-96. 
41. Mehra A, Baker CL, Loros JJ, et al. Post-translational modifications in circadian rhythms. Trends Biochem Sci. 2009 Oct;34(10):483-90.

42. Cavadini G, Petrzilka S, Kohler P, et al. TNF-alpha suppresses the expression of clock genes by interfering with E-box-mediated transcription. Proc Natl Acad Sci U S A. 2007 Jul 31;104(31):12843-8.

43. Hashiramoto A, Yamane T, Tsumiyama K, et al. Mammalian clock gene cryptochrome regulates arthritis via proinflammatory cytokine TNF-\{alpha\}. J Immunol. 2009 Dec 30.

44. Nakahata Y, Sahar S, Astarita G, et al. Circadian control of the NAD+ salvage pathway by CLOCK-SIRT1. Science. 2009 May 1;324(5927):654-7.

45. Ramsey KM, Yoshino J, Brace CS, et al. Circadian clock feedback cycle through NAMPT-mediated NAD+ biosynthesis. Science. 2009 May 1;324(5927):6514.

46. Brentano F, Schorr O, Ospelt C, et al. Pre-B cell colony-enhancing factor/visfatin, a new marker of inflammation in rheumatoid arthritis with proinflammatory and matrix-degrading activities. Arthritis Rheum. 2007 Sep;56(9):2829-39.

47. Luk T, Malam Z, Marshall JC. Pre-B cell colony-enhancing factor (PBEF)/visfatin: a novel mediator of innate immunity. J Leukoc Biol. 2008 Apr;83(4):804-16.

48. Courties G, Brentano F, Niederer F, et al. RNA interference-mediated gene therapy of PBEF reduces the severity of collagen-induced arthritis in mice. Ann Rheum Dis. 2009;Vol 68 (Suppl3):194.

49. Yamakuchi M, Lowenstein CJ. MiR-34, SIRT1 and p53: the feedback loop. Cell Cycle. 2009 Mar 1;8(5):712-5. 
50. Niederer F, Brentano F, Ospelt C, et al. Expression of sirtuins in rheumatoid arthritis synovial fibroblasts. Arthritis Rheum., 2009; 60,(10):S52.

51. Yeung F, Hoberg JE, Ramsey CS, et al. Modulation of NF-kappaB-dependent transcription and cell survival by the SIRT1 deacetylase. Embo J. 2004 Jun $16 ; 23(12): 2369-80$.

52. Salminen A, Kaarniranta K. SIRT1: Regulation of longevity via autophagy. Cell Signal. 2009 Feb 26.

53. Nayagam VM, Wang $\mathrm{X}$, Tan $\mathrm{YC}$, et al. SIRT1 modulating compounds from high-throughput screening as anti-inflammatory and insulin-sensitizing agents. J Biomol Screen. 2006 Dec;11(8):959-67.

54. Yang Y, Fu W, Chen J, et al. SIRT1 sumoylation regulates its deacetylase activity and cellular response to genotoxic stress. Nat Cell Biol. 2007 Nov;9(11):125362.

55. Franz JK, Pap T, Hummel KM, et al. Expression of sentrin, a novel antiapoptotic molecule, at sites of synovial invasion in rheumatoid arthritis. Arthritis Rheum. 2000 Mar;43(3):599-607.

56. Meinecke I, Cinski A, Baier A, et al. Modification of nuclear PML protein by SUMO-1 regulates Fas-induced apoptosis in rheumatoid arthritis synovial fibroblasts. Proc Natl Acad Sci U S A. 2007 Mar 20;104(12):5073-8.

57. Langley E, Pearson M, Faretta M, et al. Human SIR2 deacetylates p53 and antagonizes PML/p53-induced cellular senescence. Embo J. 2002 May $15 ; 21(10): 2383-96$.

58. Takayama K, Ishida K, Matsushita T, et al. SIRT1 regulation of apoptosis of human chondrocytes. Arthritis Rheum. 2009 Sep;60(9):2731-40. 
59. Dvir-Ginzberg M, Gagarina V, Lee EJ, et al. Regulation of cartilage-specific gene expression in human chondrocytes by SirT1 and nicotinamide phosphoribosyltransferase. J Biol Chem. 2008 Dec 26;283(52):36300-10.

60. Kurowska-Stolarska $\mathrm{M}$, Distler $\mathrm{JH}$, Jungel $\mathrm{A}$, et al. Inhibitor of DNA binding/differentiation 2 induced by hypoxia promotes synovial fibroblast-dependent osteoclastogenesis. Arthritis Rheum. 2009 Dec;60(12):3663-75.

61. Distler JH, Wenger RH, Gassmann M, et al. Physiologic responses to hypoxia and implications for hypoxia-inducible factors in the pathogenesis of rheumatoid arthritis. Arthritis Rheum. 2004 Jan;50(1):10-23.

62. Giatromanolaki A, Sivridis E, Maltezos E, et al. Upregulated hypoxia inducible factor-1alpha and -2alpha pathway in rheumatoid arthritis and osteoarthritis. Arthritis Res Ther. 2003;5(4):R193-201.

63. Dioum EM, Chen R, Alexander MS, et al. Regulation of hypoxia-inducible factor 2alpha signaling by the stress-responsive deacetylase sirtuin 1. Science. 2009 Jun 5;324(5932):1289-93.

64. Guarente L. Cell biology. Hypoxic hookup. Science. 2009 Jun $5 ; 324(5932): 1281-2$

65. Potente M, Ghaeni L, Baldessari D, et al. SIRT1 controls endothelial angiogenic functions during vascular growth. Genes Dev. 2007 Oct 15;21(20):264458.

66. Potente M, Dimmeler S. Emerging roles of SIRT1 in vascular endothelial homeostasis. Cell Cycle. 2008 Jul 15;7(14):2117-22.

67. Westerweel PE, Verhaar MC. Endothelial progenitor cell dysfunction in rheumatic disease. Nat Rev Rheumatol. 2009 Jun;5(6):332-40.

68. Brandes RP. Activating SIRT1: a new strategy to prevent atherosclerosis? Cardiovasc Res. 2008 Nov 1;80(2):163-4. 
69. Menghini R, Casagrande V, Cardellini M, et al. MicroRNA 217 modulates endothelial cell senescence via silent information regulator 1. Circulation. 2009 Oct 13;120(15):1524-32.

70. Ouyang J, Gill G. SUMO engages multiple corepressors to regulate chromatin structure and transcription. Epigenetics. 2009 Oct;4(7):440-4.

71. Maciejewska-Rodrigues H, Karouzakis E, Strietholt S, et al. Epigenetics and Rheumatoid Arthritis: The role of SENP1 in the regulation of MMP-1 expression J Autoimmun. 2010;in press.

72. Zhao S, Long H, Lu Q. Epigenetic perspectives in systemic lupus erythematosus: Pathogenesis, biomarkers, and therapeutic potentials. Clin Rev Allergy Immunol. 2009 Jul 29;Epub ahead of print.

73. Javierre BM, Fernandez AF, Richter J, et al. Changes in the pattern of DNA methylation associate with twin discordance in systemic lupus erythematosus. Genome Res. 2009 Dec 22;Epub ahead of print

74. Karouzakis E, Gay RE, Michel BA, et al. DNA hypomethylation in rheumatoid arthritis synovial fibroblasts. Arthritis Rheum. 2009 Dec;60(12):3613-22.

75. Karouzakis E, Gay RE, Gay S, et al. Epigenetic control in rheumatoid arthritis synovial fibroblasts. Nat Rev Rheumatol. 2009 May;5(5):266-72.

76. Liu Y, Chen Y, Richardson B. Decreased DNA methyltransferase levels contribute to abnormal gene expression in "senescent" CD4(+)CD28(-) T cells. Clin Immunol. 2009 Aug;132(2):257-65.

77. Griffiths EA, Gore SD. MicroRNA: mIR-ly regulators of DNMT? Blood. 2009 Jun 18;113(25):6269-70.

78. Garzon R, Liu S, Fabbri M, et al. MicroRNA-29b induces global DNA hypomethylation and tumor suppressor gene reexpression in acute myeloid leukemia 
by targeting directly DNMT3A and 3B and indirectly DNMT1. Blood. 2009 Jun 18;113(25):6411-8.

79. Chuang JC, Jones PA. Epigenetics and microRNAs. Pediatr Res. 2007 May;61(5 Pt 2):24R-9R.

80. Stanczyk J, Pedrioli DM, Brentano F, et al. Altered expression of MicroRNA in synovial fibroblasts and synovial tissue in rheumatoid arthritis. Arthritis Rheum. 2008 Apr;58(4):1001-9.

81. Nakasa T, Miyaki S, Okubo A, et al. Expression of microRNA-146 in rheumatoid arthritis synovial tissue. Arthritis Rheum. 2008 May;58(5):1284-92.

82. Stanczyk Feldges J, Karouzakis E, Jüngel A, et al. Mir-203 regulates the expression of IL-6 and matrixmetalloproteinase (MMP)-1 in RA synovial fibroblasts. Arthritis Rheum., 2009;60, 10, S706.

83. Nakamachi $\mathrm{Y}$, Kawano $\mathrm{S}$, Takenokuchi M, et al. MicroRNA-124a is a key regulator of proliferation and monocyte chemoattractant protein 1 secretion in fibroblast-like synoviocytes from patients with rheumatoid arthritis. Arthritis Rheum. 2009 May;60(5):1294-304.

84. Alsaleh G, Suffert G, Semaan N, et al. Bruton's tyrosine kinase is involved in miR-346-related regulation of IL-18 release by lipopolysaccharide-activated rheumatoid fibroblast-like synoviocytes. J Immunol. 2009 Apr 15;182(8):5088-97.

85. Nagata $\mathrm{Y}$, Nakasa $\mathrm{T}$, Mochizuki $\mathrm{Y}$, et al. Induction of apoptosis in the synovium of mice with autoantibody-mediated arthritis by the intraarticular injection of double-stranded MicroRNA-15a. Arthritis Rheum. 2009 Sep;60(9):2677-83. 